\title{
Visualization of Single Molecules Building a Viral Capsid Protein Lattice through Stochastic Pathways
}

\author{
Alejandro Valbuena, ${ }^{\S}$ Sourav Maity, ${ }^{\S}$ Mauricio G. Mateu,* and Wouter H. Roos*
}

Cite This: ACS Nano 2020, 14, 8724-8734

Read Online

ABSTRACT: Direct visualization of pathways followed by single molecules while they spontaneously self-assemble into supramolecular biological machines may provide fundamental knowledge to guide molecular therapeutics and the bottom-up design of nanomaterials and nanodevices. Here, high-speed atomic force microscopy is used to visualize self-assembly of the bidimensional lattice of protein molecules that constitutes the framework of the mature human immunodeficiency virus Jigsaw puzzle-like building of a virus capsid lattice

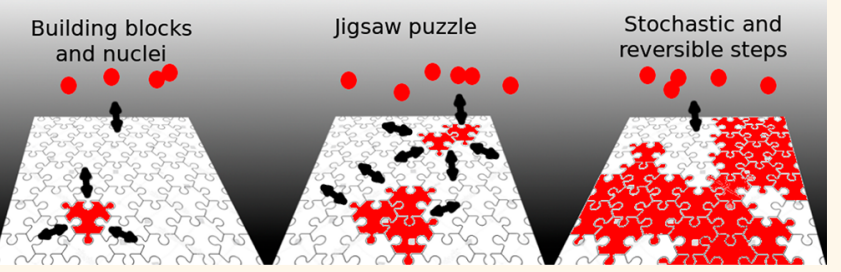
capsid. By real-time imaging of the assembly reaction, individual transient intermediates and reaction pathways followed by single molecules could be revealed. As when assembling a jigsaw puzzle, the capsid protein lattice is randomly built. Lattice patches grow independently from separate nucleation events whereby individual molecules follow different paths. Protein subunits can be added individually, while others form oligomers before joining a lattice or are occasionally removed from the latter. Direct real-time imaging of supramolecular selfassembly has revealed a complex, chaotic process involving multiple routes followed by individual molecules that are inaccessible to bulk (averaging) techniques.

KEYWORDS: self-assembly, nanomaterials, virus, capsid protein, single-molecule, high-speed atomic force microscopy

$\mathrm{T}$ he self-assembly of many biomolecular machines including viruses and viral capsids ${ }^{1-3}$ from their molecular building blocks is a complex, yet surprisingly faithful and efficient process that has fascinated biologists, chemists, physicists, engineers, nanoscientists, and nanotechnologists alike. As a consequence, many multidisciplinary studies on the assembly or disassembly of virus particles have been undertaken and have been reviewed. ${ }^{4-10}$ Such experimental and theoretical studies are contributing to understanding supramolecular self-assembly and its relationship to biological function; ${ }^{4-10}$ the development of antiviral drugs that inhibit or misdirect virus morphogenesis or genome uncoating; $^{11,12}$ the engineering of virus-like nanoparticles and nanomaterials for biomedical or nanotechnological applications; ${ }^{13-16}$ and the design and fabrication using a bottom-up approach of other nanostructures that can self-assemble in one, two, or three dimensions. ${ }^{17-20}$

Understanding the high fidelity and efficiency of the supramolecular self-assembly of proteins and other biomolecules requires a deep knowledge not only of specific molecular interactions and thermodynamic parameters but also of the individual pathways followed by individual molecules during the process. The information needed for folding and binding of the protein molecules that build most biological complexes is entirely encoded in their three-dimensional structure. ${ }^{21}$ Inside the cell, protein folding or virus assembly may require the action of chaperones or other ancillary factors to minimize offpathway intermediates and kinetic traps under a complex, macromolecularly crowded environment. ${ }^{21}$ However, under adequate conditions authentic protein complexes and virus capsids can be efficiently and faithfully assembled in vitro in the absence of any other component. For example, the capsid protein of human immunodeficiency virus alone can form assemblies that are virtually indistinguishable in shape and molecular architecture from authentic mature virus capsids. $^{22-25}$ This fact has allowed the study of fundamental protein or virus assembly reactions using simplified theoretical models and/or minimalist experimental systems, without a need to reproduce the complexity of the intracellular environment.

Received: April 16, 2020

Accepted: June 26, 2020

Published: July 7, 2020 
Theoretical models and simulations indicate that pathways for folding and assembly of protein and protein assemblies proceed downhill through "folding funnels" in an energy landscape, ${ }^{21}$ and both simplified models and coarse-grained (CG) simulations have predicted reaction pathways during self-assembly of virus-like particles. ${ }^{4-6,8-10,26}$ Unfortunately, experimental studies using actual virus capsids or virions to verify those predictions have frequently met with difficulties, mainly due to the highly transient nature and possible heterogeneity of most reaction intermediates.

Remarkable progress has, however, been made to experimentally identify populated intermediates along the assembly or disassembly pathways of different virus particles. ${ }^{4,5,7,27}$ In many cases intermediates were stabilized relative to initial and/ or final states through changes in conditions or genetic modifications in the viral proteins. A number of those intermediates were characterized by different techniques including electron microscopy (EM), ${ }^{28-31}$ biochemical and genetic analyses, ${ }^{32}$ size-exclusion chromatography, ${ }^{31}$ smallangle X-ray scattering, ${ }^{33-38}$ mass spectrometry, ${ }^{39-42}$ resistivepulse sensing, ${ }^{31,33}$ nanofluidics, ${ }^{43}$ interferometric scattering microscopy, ${ }^{44}$ fluorescence correlation spectroscopy (FCS), ${ }^{45}$ or conventional atomic force microscopy (AFM). ${ }^{3}$

Despite crucial advances in this field, the results of most experimental studies on virus assembly pathways so far only yielded a few snapshots of the whole process, limiting the verification and further development of models and simulations. Most importantly, different pathways that may be followed by individual molecules are invisible to the averaging (bulk) techniques used in most of those studies. Studies at the single-molecule level can provide novel, deeper insights into virus assembly mechanisms. An example of such an approach is the recent investigation of the formation of rod-shaped viruslike particles (VLPs) from a de novo-designed artificial polypeptide $^{46}$ and of sphere-like VLPs from SV40 capsid proteins. ${ }^{47}$ In these studies, VLP assembly around a DNA template was followed at the single-molecule level using conventional and fluorescent optical tweezers. Other singleparticle approaches include single-molecule FCS to follow encapsidation of a viral genome ${ }^{27,45}$ and scattering microscopy to study MS2 assembly at the single particle level. ${ }^{44}$

In the past decade high-speed AFM (HS-AFM) ${ }^{48}$ has emerged as a powerful single-molecule technique to visualize and characterize in real time and at the single-molecule level the dynamics of molecular complexes involved in biological processes. Working molecular motors, ${ }^{49,50}$ movement of membrane-associated proteins, ${ }^{51-53}$ and the dynamics of macromolecular complexes ${ }^{54-57}$ are increasingly being visualized and analyzed using HS-AFM in liquid under close to physiological conditions. In comparison with conventional AFM, HS-AFM is capable of acquiring images at least 100 times faster, allowing to reach up to video rate temporal resolution. Moreover, the fast scanning rates used in HS-AFM allow imaging of highly mobile and often flexible and soft biological samples with a spatial resolution higher than that achieved at the much lower scanning rates of conventional AFM where the fast moving moieties are smeared out. In comparison with other single-molecule techniques such as optical tweezers, FCS, or interferometry, the strength of HSAFM is to allow visualization of every single molecule and every transient intermediate, even if largely unpopulated, with high spatial and temporal resolution.
The hexagonal protein lattice that constitutes the framework of the mature capsid in human immunodeficiency virus $(\mathrm{HIV})^{22,23}$ was used here as a model to visualize by HSAFM the trajectories followed by single molecules, while they build a supramolecular viral protein network. The mature HIV capsid protein (CA) monomer is made of two domains (NTD and CTD) connected by a flexible linker (Figure 1A, top left).

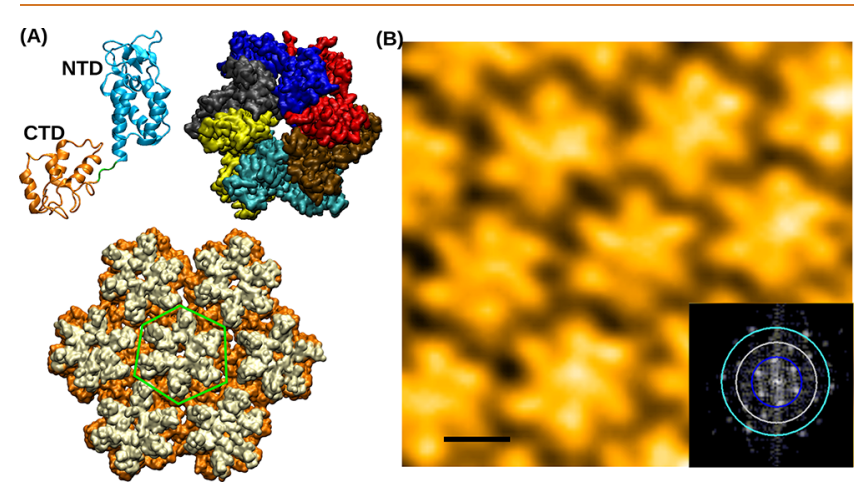

Figure 1. The mature HIV capsid protein lattice. (A) Top left: ribbon diagram of a CA monomer. NTD (cyan) and CTD (orange) domains are linked by a hinge region (green). Top right: atomic surface model of a CA hexamer. The 6 CA monomers are shown in different colors. Bottom: atomic surface model of a part of the mature HIV capsid protein lattice formed by 7 bound CA hexamers. The interface between the central hexamer and neighboring hexamers is indicated by a green line. Atomic coordinates were taken from the Protein Data Bank (PDB ID: 3J3Y). (B) HS-AFM single image of a small area of the mature HIV capsid protein lattice on a flat surface. Scale bar is $5 \mathrm{~nm}$. The inset corresponds to the Fast Fourier Transformed (FFT) representation of the image. The concentric circles localize different lattice vector values $(9,5$, and $4 \mathrm{~nm})$.

Initially, a bidimensional (2D) CA lattice ${ }^{58}$ is formed by CA hexamers stabilized by NTD-NTD and NTD-CTD interactions (Figure 1A, top right) and bound to neighboring hexamers through dimeric CTD-CTD interactions (Figure 1A, bottom). The growing hexagonal lattice made of CA proteins (termed here the capsid protein lattice) surrounds the viral ribonucleoprotein (RNP) complex, while it folds in the third dimension to yield a closed, truncated cone-shaped capsid made by only a fraction $(\sim 1000-1500)$ of the $\sim 2500 \mathrm{CA}$ monomers contained in the virion. ${ }^{22}$ Capsid closure and conical shape result from both lattice curvature and the introduction of CA pentamer "defects" at certain positions in the hexagonal lattice. ${ }^{22,23}$ At high ionic strength or in the presence of macromolecular crowding agents, free CA from HIV can polymerize in vitro ${ }^{59,60}$ as hollow tubes or flat sheets based on the same hexagonal lattice present in the authentic capsid but (at least theoretically) without pentagonal defects. Under certain conditions in in vitro experiments closed, coneshaped capsid-like particles that must include CA pentamers were abundantly formed. ${ }^{24}$

Using conventional AFM we had previously shown ${ }^{61,62}$ that $\mathrm{CA}$ at near physiological temperature, $\mathrm{pH}$, and ionic strength and in the absence of other macromolecules can efficiently selfassociate as a monomolecular layer on a flat mica substrate that acts as an assembly promoting template. The mica surface has a considerable negative charge density and may provide a $2 \mathrm{D}$ qualitative electrostatic mimic of the tridimensional (3D) RNP complex in the mature virion, which is also negatively charged 


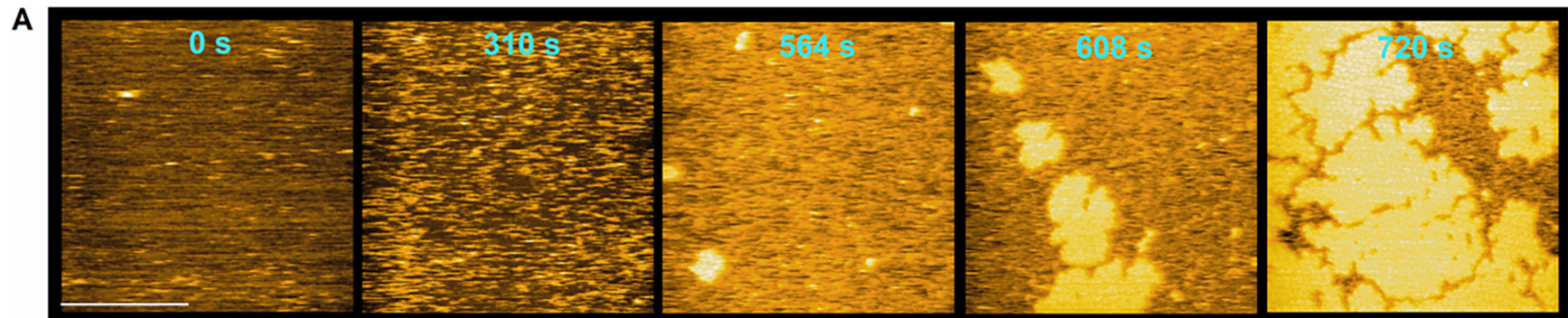

B

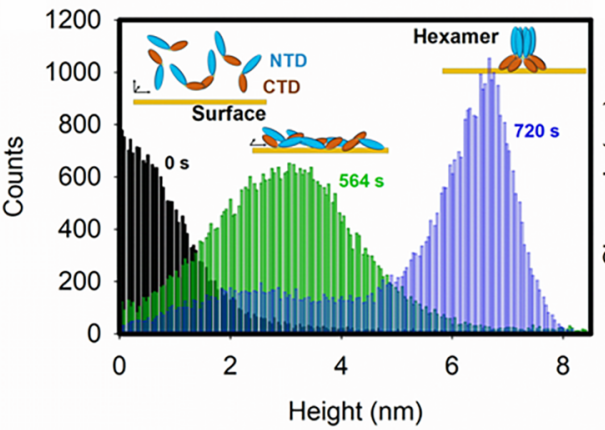

E

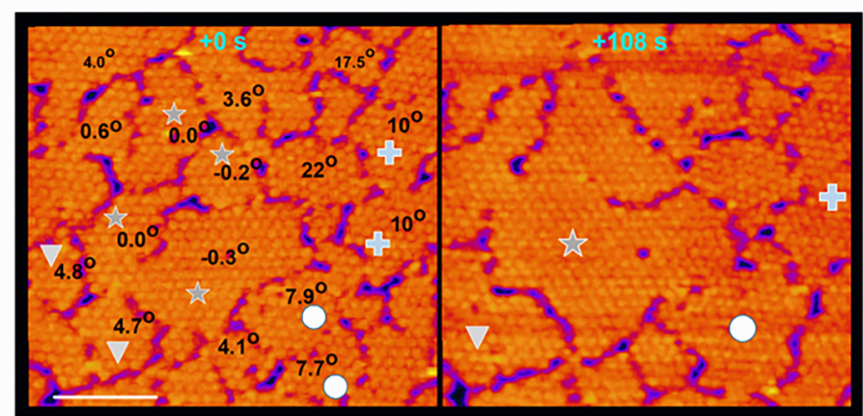

C

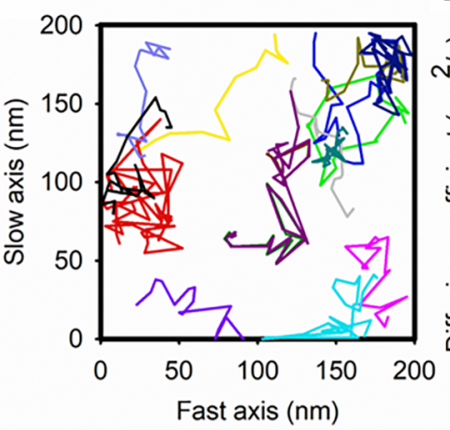

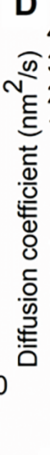

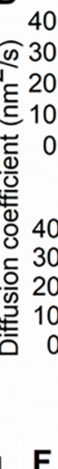

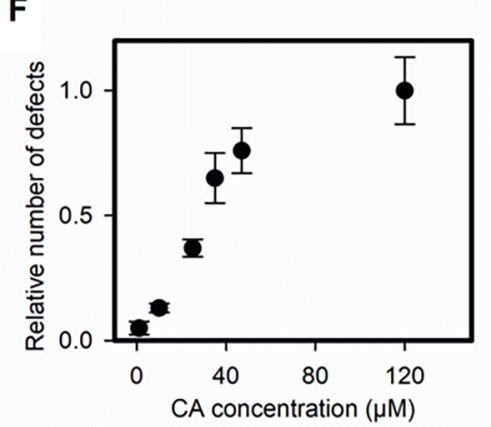

Figure 2. Nucleation, diffusion, growth, and fusion during assembly of the HIV capsid protein lattice. (A) Representative frames from Movie 2 (rate $0.5 \mathrm{fps}$ ) at the times indicated in each frame. [CA] $=10 \mu \mathrm{M}$. Scale bar (first frame, bottom) is $100 \mathrm{~nm}$. (B) Height histograms calculated from AFM images taken at different times from Movie 2. Different colors correspond to height profiles determined at different times as indicated in the plot. The three insets depict likely molecular arrangements that may correspond to peaks of different heights $(\sim 1-$ $2 \mathrm{~nm}, \sim 2.5 \mathrm{~nm}, \sim 6.5 \mathrm{~nm}$ ) in the histogram (see text). (C) Trajectories (each shown in a different color) of individual small oligomers on the substrate before they became immobilized. (D) top panel, Average diffusion coefficient for oligomers (black dots and fitting line) and percent area occupied by the protein lattice (green line) as a function of time. bottom panel, Average diffusion coefficient for oligomers as a function of surface occupancy. (E) Two time-lapse AFM images ( 0 and $108 \mathrm{~s}$ ) showing the importance of relative lattice axial angles for the fusion of converging lattice patches. Left image reports the lattice angles for individual patches; the right image shows the merger of patches with a similar angle and the generation of defects for the unmatched patches. Different symbols provide a guide for lattice integration when left and right images are compared. (F) Relative number of defects per $1 \mu \mathrm{m}^{2}$ imaging area detected after $3 \mathrm{~h}$ of incubation, as a function of CA concentration.

mainly due to RNA phosphates. ${ }^{61}$ The architecture of the CA lattice assembled on mica was similar to the hexagonal $\mathrm{CA}$ lattice in HIV capsid-like particles assembled in solution in vitro or in the authentic mature HIV capsid.

In the present study, HS-AFM has been used to visualize in real time transient intermediates and individual reaction pathways followed by single molecules during templated selfassembly of a flat lattice made of HIV capsid protein subunits. The results provide direct experimental evidence for the concept that the reversible self-assembly of a protein lattice from its building blocks does not proceed through a predominant pathway. Instead, resembling how a jigsaw puzzle is built, self-assembly of a $2 \mathrm{D}$ hexagonal lattice of a viral capsid protein is a complex stochastic process involving multiple, different routes followed by single molecules. Up to now this feature had remained obscure as the followed routes are not accessible to bulk, averaging experiments.

\section{RESULTS AND DISCUSSION}

\section{HIV Capsid Protein Lattice Architecture Visualized by} Resolving Individual Protein Subunits Using HS-AFM. The self-assembled HIV capsid protein lattice was first imaged by HS-AFM in order to determine structural features of the lattice (Figure 1B and Movie 1). Because of the orientation adopted by each CA protein in the lattice on the mica substrate, with the NTD on top and the CTD below, ${ }^{61}$ only the topography of the NTDs can be probed by the AFM tip. The spatial resolution achieved was high enough to reveal the location of individual NTDs in the lattice and the particular shape of individual hexamers. Precise measurements of individual distances and angles between lattice elements were 
obtained, and the Fourier transform of the lattice revealed the regularity of the assembly (Figures 1B-inset and S1). The average values of the lattice parameters (Figure S1) are in agreement with those previously obtained from static images obtained using other techniques such as cryo-EM, ${ }^{23}$ electron cryocrystallography, ${ }^{58}$ and conventional AFM. ${ }^{61}$ In a closely packed hexagonal lattice, each monomer forms an angle of $\pm 30^{\circ}$ with the lattice axis, whereas the NTD domains in the CA lattice are oriented with an angle of either $\pm 20^{\circ}$ or $\pm 40^{\circ}$, as can be observed in the images (Figures S1B and S1G). The high spatial resolution achieved by HS-AFM in time-resolved experiments described next allowed us to distinguish between partially or fully assembled hexamers and to visualize in real time and trace individual pathways for association or dissociation of CA monomers or small oligomers during selfassembly or disassembly of the HIV capsid protein lattice.

Dynamics of Nucleation Points and Growth during Self-Assembly of an HIV Capsid-Based Protein Lattice. Self-assembly of the viral capsid protein lattice on the negatively charged mica surface was initiated by adding free CA protein to the buffer solution (PBS) in the AFM liquid chamber and was monitored at high temporal resolution (0.52 frames per second, fps) by HS-AFM imaging. At $[\mathrm{CA}] \geq 10$ $\mu \mathrm{M}$ typical stages in the formation of a protein complex (lag phase, nucleation, growth, and saturation) were observed as specific changes in surface topography and protein-covered area (Movie 2 and Figures 2 and S2). The surface gradually changed its topography as free CA molecules interacted both with the substrate and between them. As the molecular density on the surface increased, small oligomers were detected that moved on the surface until they were immobilized and started to grow into larger patches (Movie 2 and Figure 2). Height distributions traced at different times indicate a clear succession of distinguishable molecular arrangements (Figures $2 \mathrm{~B}$ and S2A, S2B). The initial unperturbed surface at $t=0 \mathrm{~s}$ started to exhibit many structural elements of increased height over time, as can be seen from the height distribution at $t=$ $310 \mathrm{~s}$. The height distribution obtained at $t=564 \mathrm{~s}$, when the surface was already largely covered by a fluctuating array of crowded CA molecules, revealed a dominant peak of elements $\sim 2.5 \mathrm{~nm}$ in height. At $t=608 \mathrm{~s}$ as lattice patches were growing, a new peak of elements $\sim 6.5 \mathrm{~nm}$ in height started to be observed, and this peak became dominant at $t=720 \mathrm{~s}$ when the ordered CA lattice covered most of the surface (Figures 2B and S2B, S2C). These observations suggest the following succession of early assembly events (Figure S2A): prior to lattice formation, free CA molecules (step 1) diffuse on the substrate, using a maximum of their molecular surface area to contact the substrate (i.e., laying down) (step 2), and quickly cover the available surface (step 3). Later, more complex structural elements (small oligomers) are formed standing up on the substrate (step 4), and additional CA elements are merged with each other through lateral association (step 5), leading to growth of the lattice patches.

Early small oligomers had a size compatible with those of a CA hexamer or a trimer of dimers (height $\sim 6.5 \mathrm{~nm}$ ). Detailed analysis of their individual in-plane trajectories (Figure $2 \mathrm{C}$ and Movie 2) revealed their random isotropic movements (Figure S3) on the surface. Their average diffusion coefficient was gradually reduced as a function of both time and increasing surface occupancy by the proteins (Figure 2D). These small oligomers did not grow into larger protein patches until they became immobilized. They then either acted as nucleation points and grew into larger lattice patches by incorporating additional CA subunits or associated with an existing patch (Movie 2 and Figure 2A). Interestingly, those oligomers were not generally observed before the surface area covered by CA reached a certain threshold $(56 \% \pm 10 \%$ in these experiments), which was independent of total [CA] (Figure S2C). This observation suggests that a critical protein density on the surface is required for the phase transition (precursor $\leftrightarrow$ nucleus) during capsid protein lattice assembly.

The growth of several protein patches that had been formed from individual nuclei was then analyzed (Movie 2 and Figure S4). Under the conditions used in these experiments, the growth rate, measured in terms of protein-covered area (Figure S4B, black triangles), increased linearly at $\sim 190 \mathrm{~nm}^{2} / \mathrm{s}$ until $t$ $\approx 150 \mathrm{~s}$, and the same was observed for the patch perimeter (Figure S4C, black dots), after which the surface became saturated. Fluctuations in lattice surface area $(\Delta A)$ (Figure S4B, red dots-cyan trace) or perimeter (Figure S4C, red dotsgreen trace) during the first $150 \mathrm{~s}$ indicate that assembly and disassembly events were both taking place, while keeping a net positive growth rate. Moreover, a fluctuation in perimeter (Figure S4C, red dots-green trace) also indicates, as the lattice expands further, that a spontaneous rearrangement in the lattice circumference takes place. This kind of growth allowed any particular protein patch to grow along any direction and to efficiently cover the underlying substrate (Movie 2 and Figure 2A).

Individual patches sometimes merged together, while others were not merged (Figure 2E) even after incubation times of up to $3 \mathrm{~h}$. Closer inspection suggests that neighboring lattice patches with the same orientation tend to merge together (Figure 2E), while lattice patches with substantially different orientations may not be merged, leading to "defects" in the coverage of the substrate between merged lattice patches. It was also found that the number of defects increased with increasing total $[\mathrm{CA}]$ until it reached a saturation point at the highest tested [CA] (Figure 2F).

Visualization in Real Time by HS-AFM of Multiple Pathways for Building New Hexamers during Stochastic Growth of an HIV Capsid-Based Protein Lattice. In order to reveal individual transient intermediates and reaction pathways for assembly of the viral capsid protein lattice, the dynamics of single protein molecules during growth of the CA lattice was visualized by HS-AFM. Detailed analysis of the high-resolution videos revealed the successive association to growing capsid protein lattice patches of individual small-sized molecular components (such as CA monomers or dimers) interspersed with temporary dissociation of other individual components (Movies 3 and 4, Figures 3 and S5).

Movie 3 and Figure 3A exemplify the gradual assembly of a new hexamer at the capsid protein lattice edge. Here single CA subunits associate in a clockwise manner around the hexamer 6-fold symmetry axis. At $0.5 \mathrm{~s}$, an empty space can be observed where the new hexamer will be assembled. Three CA subunits were first bound at the lattice edge in this location (image at $1.5 \mathrm{~s})$; then a single CA subunit was removed (5.5 s); and another CA subunit was added later $(6.5 \mathrm{~s})$, followed by a fourth $(7.5 \mathrm{~s})$, a fifth $(10.5 \mathrm{~s})$, and a sixth subunit $(11.5 \mathrm{~s})$.

Lattice growth that included dynamic rearrangements of capsid protein lattice components was also observed. Movie 4 and Figure $3 \mathrm{~B}$ show the way in which three new hexamers were gradually assembled at the edge of the capsid protein lattice. Two of these hexamers assembled only after the CA 


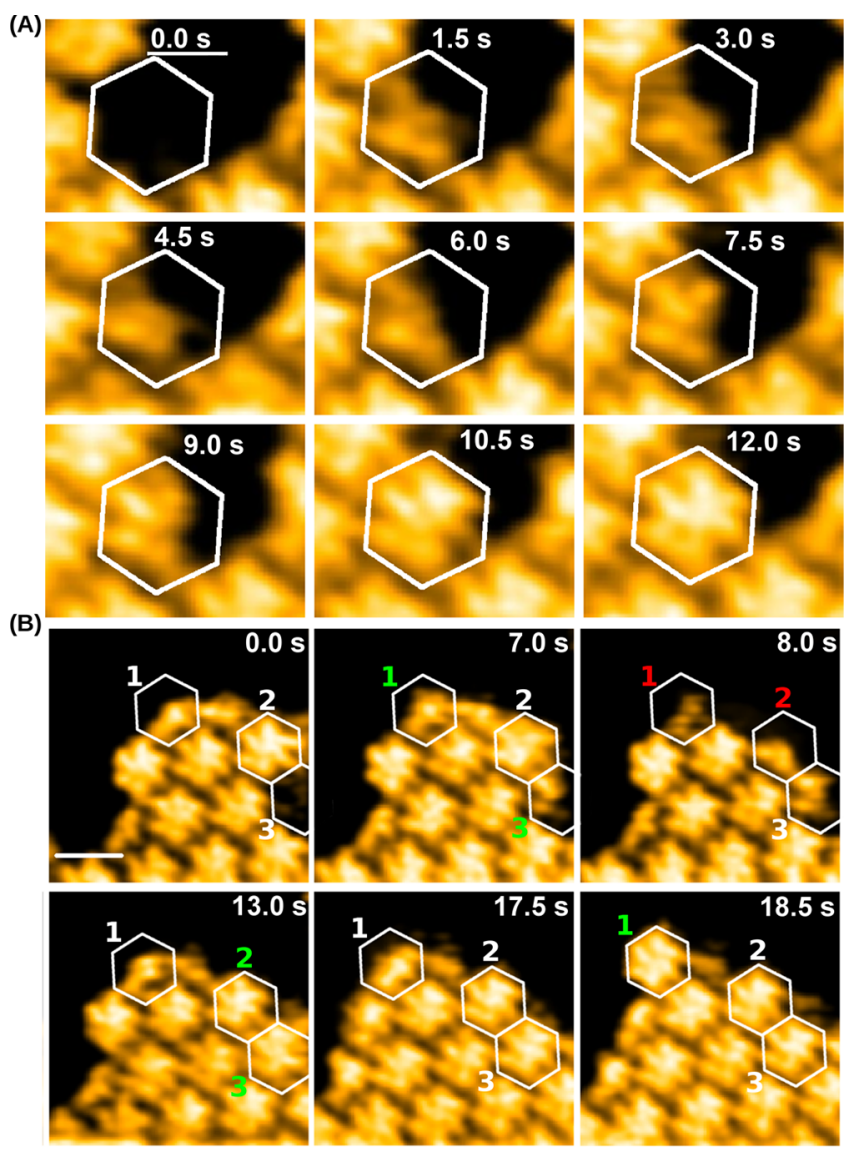

Figure 3. Dynamics of self-assembly of the HIV capsid protein lattice visualized by HS-AFM. Representative frames from Movie 3 (A) or Movie 4 (B) (rate 2 fps) are shown. In (A) a white hexagon marks the position where a hexamer at the edge of the growing capsid protein lattice will be gradually assembled from CA monomers, dimers, or smaller oligomers (see text and Movie 3). $[\mathrm{CA}]=20 \mu \mathrm{M}$. In (B) three white hexagons (numbered 1, 2, and 3 ) are used to denote locations at the edge of the capsid protein lattice where individual hexamers are being gradually assembled and/or disassembled through different pathways. Color coding of those numbers $(1,2$, and 3$)$ in each frame denotes association events (green number), a dissociation event (red number), or no event (white number) taking place at the labeled location (white hexagon) in each defined time step (video frame) (see text and Movie 4). Scale bar (white line in panel B, first frame) is $10 \mathrm{~nm}$. The same scale was used in both panels (A and B).

subunits corresponding to one partially formed hexamer and another fully formed hexamer pre-existent at these same positions dissociated from the lattice.

In other experiments, the balance between assembly and disassembly events was altered in order to favor the net disassembly of a previously assembled capsid protein lattice. This bias toward disassembly can be achieved by a combination of two factors: limited amounts of free CA protein and deliberately elevated imaging forces on the assembled lattice. The experiments revealed that hexamers were gradually removed from the lattice edge by stepwise dissociation of single CA monomers or small CA oligomers (Movie 5 and Figure S6A). Occasionally, transient clusters of hexamer-sized oligomers were observed that eventually dissociated into free hexamer-sized oligomers that could be followed for relatively long times. These hexamer-sized oligomers had the capacity to bind again to the lattice edge.
For instance, a free hexamer-sized oligomer remained visible for at least $\sim 25 \mathrm{~s}$ before it reassociated with the protein lattice (Movie 6 and Figure S6B). These observations show that, in addition to $\mathrm{CA}$ monomers or smaller oligomers, integral $\mathrm{CA}$ hexamers or trimers of CA dimers can be added to or removed from the capsid protein lattice as integral components.

Representative trajectories leading to assembly of individual hexamers at either $10 \mu \mathrm{M}$ or $20 \mu \mathrm{M}$ total [CA] are compared in Figure 4A. In every case, CA subunits were incorporated
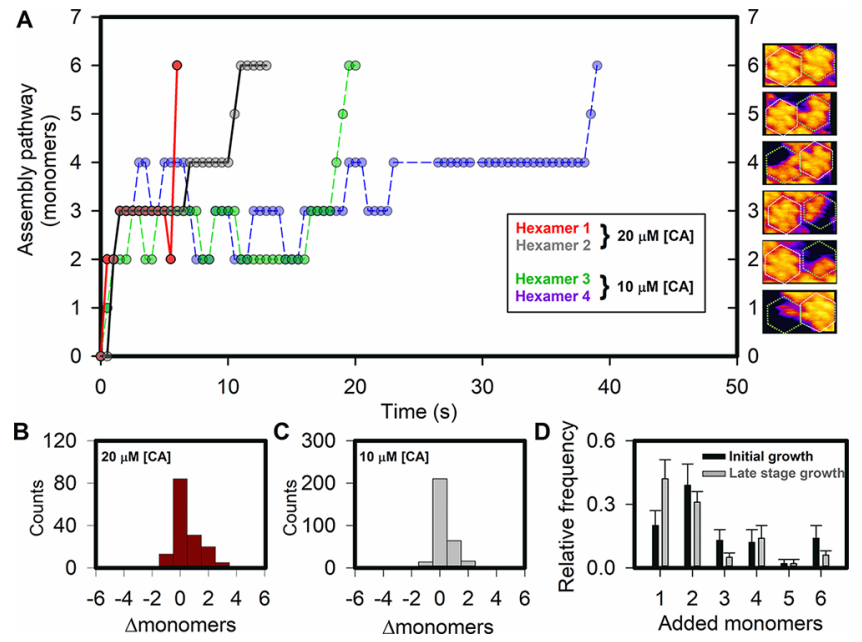

Figure 4. Stochastic pathways for the formation of individual hexamers during self-assembly of the HIV capsid protein lattice. (A) Representative assembly pathways of 4 individual hexamers, at $10 \mu \mathrm{M}$ total [CA] (green/blue dots and dashed traces) and at 20 $\mu \mathrm{M}$ (red/gray dots and solid traces). Insets showing examples of images depicting the number of monomers in an incomplete hexamer next to a complete hexamer. (B) and (C) Number of frames in which a given number of CA monomers are added to (positive values) or removed from (negative values) an assembling hexamer in the capsid protein lattice. Total $[\mathrm{CA}]$ was $20 \mu \mathrm{M}$ (B) or $10 \mu \mathrm{M}(\mathrm{C})$, and assembly of 15 (B) or 11 (C) hexamers was monitored. (D) Relative frequency with which different numbers of monomers ( 1 to 6 ) were added to the lattice in single time steps, either during the initial growth (black bars) or at a late stage with the surface nearly fully covered (gray bars).

into the growing hexamer in a stochastic process in which both association and dissociation events, separated by variable intervals, were observed (Figure 4A). Trajectory analysis was used to determine the number of CA subunits added in each time step (typically $0.5 \mathrm{~s}$ ) during the gradual formation of new hexamers at the edge of the capsid protein lattice. A variety of different trajectories were observed when individual cases were examined, and differences were also detected when different total [CA] were used (Figure 4). At $20 \mu \mathrm{M}$, in each time step the binding of $0,1,2$, or 3 subunits was observed (Figure 4B). At $10 \mu \mathrm{M}$, the binding of 0,1 , or 2 (but not 3 ) subunits was observed (Figure 4C). Irrespective of the protein concentration used, in most time steps the hexamer being formed remained in a resting state with no subunits added or removed (Figure 4B,C).

Analysis of the relative frequencies with which a different number of subunits were added to a growing hexamer at the lattice edge per time step revealed that during early growth of capsid protein lattice patches addition of two subunits was more probable than addition of only one subunit (Figure 4D, black bars); while at later stages of lattice growth, closer to 
reaching equilibrium between association and dissociation events (Figure S7), addition of one subunit only was more probable than addition of two subunits (Figure 4D, white bars). The average time for assembly of a new single hexamer at the lattice edge (Figure 4A) was also dependent on the progress of the assembly reaction (Figure 4D) and the remaining available accessible area. To sum up, templated growth of the HIV capsid protein lattice proceeded by the random, stepwise addition to the lattice edge of CA monomers or small oligomers, interspersed with dissociation of some of these elements in a reversible, stochastic process.

Experimental Evidence Directly Reveals That the Dynamics of Supramolecular Self-Assembly of a Viral Protein Lattice Resembles That of Jigsaw PuzzleBuilding. Both theoretical models and simulations and experimental studies have revealed that self-assembly of both helical and icosahedral virus capsids can be generally described as a reversible nucleation and growth process. $^{4-10,38,63,64}$ Nucleation and growth is also a feature of mature HIV CA assembly into a capsid-like hexagonal lattice as indicated by (i) a kinetic model ${ }^{65}$ and CG simulations ${ }^{66-70}$ and (ii) in vitro kinetics of CA self-assembly, either in 3D as hollow tubes in solution $^{59,60}$ or in $2 \mathrm{D}$ as a flat layer on a template. ${ }^{61,62}$ This latter assembly system was used here to visualize in real time by HS-AFM highly transient association and dissociation events involving individual molecules during nucleation and growth of a virus-based protein lattice. The results can be integrated into an empirical model at the single molecule level that is discussed next.

At the total $[\mathrm{CA}]$ used here $(\leq 20 \mu \mathrm{M})$, and considering the dissociation constant of the CA dimer-monomer equilibrium under similar conditions $(\sim 18 \mu \mathrm{M}),{ }^{71}$ we expect to have both monomers and dimers in solution where CA monomers will be more abundant than dimers. Both the monomers and the dimers can adsorb onto the mica substrate, partly based on electrostatic interactions between the negatively charged substrate and the positively charged CA-CTDs. The limited substrate surface area available for adsorption of the many free CA molecules present in solution eventually leads to crowded conditions on the substrate. Both CA adsorption and surface crowding likely promote, even at low total [CA] and physiological ionic strength, the observed efficient nucleation and growth of the CA lattice.

Once immobilized, early formed small oligomers can act as transient assembly nuclei as predicted by CG simulations. ${ }^{66-70}$ The very high density of free CA molecules at the beginning of the reaction did not allow the unambiguous identification of the quaternary structure of those transient oligomers. However, their size (width and height) is compatible with those of a hexamer of CA monomers or a trimer of CA dimers. $^{72}$

High-resolution HS-AFM imaging was possible when several independent CA lattice patches were already growing from oligomeric nuclei. Key observations during capsid protein lattice growth are as follows: (i) both incomplete and complete hexamers lining the patches were clearly visualized; (ii) within individual time steps one to four CA molecules became bound (either separately or together as a small oligomer) to adjacent positions at the edge of a CA patch; (iii) in some instances CA molecules bound a still incomplete hexamer, contributing to its completion (e.g., Figure 3B at $13 \mathrm{~s}$ ); (iv) on other occasions the incoming CA molecules became associated with a complete hexamer, starting the growth of a new hexamer at the lattice edge (e.g., Figure $3 \mathrm{~A}$ at $1.5 \mathrm{~s}$ ); (v) rarely, a hexamersized oligomer was seen binding to the edge of a CA patch (Figure S6B); vi) occasionally unbinding of one or several CA molecules (from monomers to hexamer-sized oligomers) was detected, even under conditions leading to net growth of the lattice (e.g., Figure 3B); and vii) the probability of addition of $\mathrm{CA}$ monomers instead of oligomers appears to increase at later times (Figure 4D).

The above key observations are consistent with a model for reversible growth of the CA lattice from nucleated patches through a succession of stochastic binding and unbinding events (Figures 3 and 5). Many of these events involve either

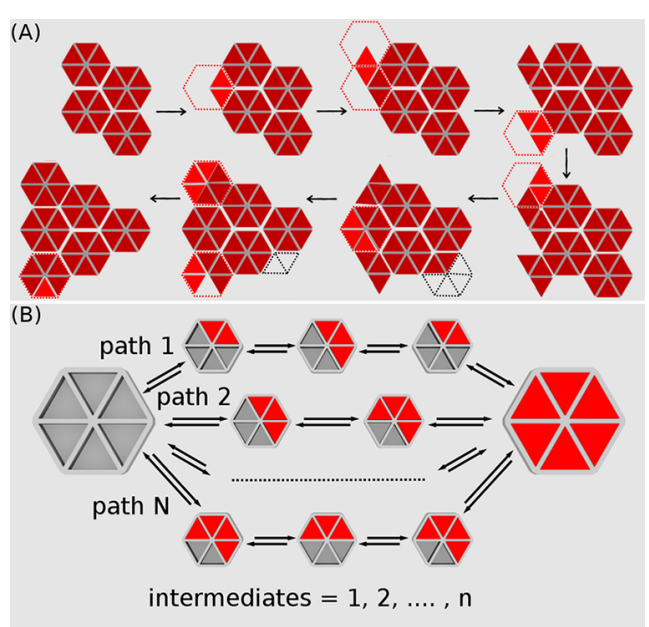

Figure 5. Stochastic, parallel pathways during assembly or disassembly of the HIV capsid protein lattice. (A) Schematic representation of different assembly or disassembly trajectories that single CA hexamers may follow during the net growth of the capsid protein lattice. For each step in the assembly reaction, CA monomers that are part of the assembled lattice are depicted in dark red; CA monomers newly bound to the edge of the lattice are shown in a lighter red hue. Red dotted lines indicate the positions of partially formed hexamers to which new CA monomers are added. CA monomers that dissociate from the lattice are represented as black dotted lines marking the original position of the unbound monomer. (B) Representation of some of the many alternative pathways during the gradual assembly (left to right arrows) of different, individual hexamers from free CA monomers or small CA oligomers that are gradually bound to the edge of the capsid protein lattice. As the process is fully reversible, the same alternative pathways may occur during the gradual disassembly of the capsid protein lattice (right to left arrows).

CA monomers or, less frequently, stable dimers (in which monomers are bound through their CTDs). Both CA monomers and dimers can bind through NTD-NTD/NTDCTD interactions to incomplete hexamers at the edge of a growing lattice patch. In addition, CA monomers (but not dimers) can bind through CTD-CTD interactions to already completed hexamers at the lattice edge. In some instances, binding or unbinding events can involve larger, transient oligomers such as trimers of CA dimers or CA hexamers or even "incomplete hexamers" (i.e., CA dimers, trimers or tetramers). In free hexamers, monomers are associated through very weak NTD-NTD/NTD-CTD interactions only, which suggest they should be highly unstable. Despite this instability, its transient formation could be facilitated by condensation on the substrate. A few of these oligomers would become stabilized by binding to the edge of a CA patch nearby. 
Instead of preformed incomplete hexamers, several independent CA monomers could be bound in rapid succession to adjacent positions at the lattice edge within the temporal resolution limit $(0.5 \mathrm{~s})$ achieved when imaging the growing CA lattice at high spatial resolution. However, frequent independent binding of 2-4 monomers at adjacent positions in such a short time seems unlikely, as there are many positions where monomers can bind and the average time between observed binding events was much longer than $0.5 \mathrm{~s}$. On the other hand, this possibility cannot be ruled out as allosteric effects may, in principle, occur ${ }^{73}$ (i.e., binding of a CA monomer to the lattice edge could favor, through some conformational rearrangement, the binding of another monomer next to it).

Two versions for lattice growth can be considered at this point, depending on the quaternary structure of the assembly nuclei. In the first version, nuclei are rather unstable CA hexamers, in which the CTDs are not bound to other CTDs. CA monomers but not dimers could bind those nuclei through CTD-CTD interactions. In the second version, nuclei are trimers of CA dimers, in which each CTD is bound to another CTD. Both CA monomers and dimers could bind those nuclei through NTD-NTD/NTD-CTD interactions.

The available evidence suggests that the first version (hexameric nuclei), though not unfeasible, is less likely than the second version (trimer-of-dimers nuclei) because of the following reasons: (i) Free hexamers in solution were not observed when a CA mutant that is unable to dimerize through its CTD was analyzed, even at very high ionic strength or under macromolecular crowding conditions that promote CA polymerization: a dimerization-competent CTD appears to be required for CA hexamerization in solution through their NTDs. $^{73}$ (ii) A kinetic model ${ }^{65}$ and CG simulations ${ }^{66-70}$ favor the existence of trimers of dimers over hexamers in solution and the role of the former as transient assembly nuclei. (iii) Formation and growth of a hexameric nucleus depend, in principle, on the availability of free CA monomers; in contrast, formation and growth of a trimer-of-dimers nucleus can be directly achieved from either CA monomers or dimers. In the conditions we used, free CA monomers must abound, but they are very scarce at the higher [CA] used in most studies of HIV capsid assembly in solution in vitro or in silico or present in the virion.

The above empirical model includes many features of surface growth, in general, and is consistent with key predictions of theoretical models and CG simulations and experimental studies of viral capsid self-assembly using mainly averaging techniques. Those features include reversibility and nucleation $^{4,5,8-10,63,64}$ and also multinucleation. ${ }^{38}$ The experimental results obtained also verify several key predictions of CG simulations of mature HIV capsid assembly in particular. ${ }^{66-70}$ The combination of high spatial and temporal resolution provided by HS-AFM confirmed such features at the single-molecule level. In addition, the HS-AFM-based visualization of self-assembly of a $2 \mathrm{D}$ protein lattice from its elementary building block (the viral CA protein) has also experimentally revealed details of a very complex, chaotic process.

The dynamics of this process resembles how a jigsaw puzzle is built. The reaction does not proceed from a single nucleus to which building blocks of a single type are successively added in monotonous succession. As when assembling a jigsaw puzzle, the $2 \mathrm{D}$ protein lattice is randomly built; several lattice patches are independently built from separate nucleation events; individual pieces of the puzzle may follow very different paths during the process; some pieces are added individually to the patches, but some others may be bound together before joining a lattice patch; the times between addition of successive pieces can be quite variable; some pieces are occasionally removed from the growing patches (either individually or in small clusters); there is the possibility that joining one piece may facilitate the rapid joining of more pieces at adjacent positions; and the patches are eventually reoriented and merged with each other. The observations do not rule out the possibility that some pathways could be favored to some extent resulting in a very complex funnel-shaped energy landscape. For example, the successive addition of CA subunits to form a new hexamer at the lattice edge seems dependent on the stage of assembly (Figure 4D). The observations of this study also provide empirical clues to develop and/or verify further precise, quantitative models and simulations of self-assembly of viral and nonviral protein lattices.

Insights into Early Capsid Assembly Steps within the Maturing HIV Virion. In this study we have used a simple model to follow the trajectories of individual protein molecules, while they build a $2 \mathrm{D}$ protein network, thus providing a single-molecule (nonaveraged) description of a supramolecular self-assembly process at high temporal resolution. In addition, these observations may provide some specific insights into the early steps of HIV capsid maturation, before later events such as the insertion of pentameric defects and capsid closure take place. $^{22}$

Maturation of the HIV virion is still a poorly understood process. $^{22}$ There are indications that it follows a mechanism in which, after proteolysis of the Gag polyprotein, the Gag-based immature capsid is fully disassembled, and the mature capsid is built from free CA building blocks. ${ }^{22}$ On the other hand, there is also data fitting with an alternative mechanism involving a phase transition between the Gag-based immature capsid and the CA-based mature capsid. ${ }^{22}$ If the immature capsid is not fully disassembled (producing free CA dimers only), ratelimiting nucleation to start mature capsid growth may not occur in the HIV virion. Irrespective of whether CA nuclei are formed at the onset of HIV maturation, our model for 2D lattice growth also describes some key features of $3 \mathrm{D}$ capsid formation in the maturing virion. In spite of quantitative differences between $2 \mathrm{D}$ and $3 \mathrm{D}$ assembly and other complications, it must be reminded that the information needed to build a viral capsid is entirely contained in the structure of its capsid building blocks. In agreement with this fundamental concept, capsid-like particles formed in vitro from CA alone can be virtually indistinguishable from authentic mature HIV capsids. ${ }^{24,25}$ Based on those considerations, capsid assembly in the maturing HIV could be expected to involve the growth of multiple CA patches through addition of individual CA monomers and small oligomers through many different, stochastic pathways.

Quantitative differences between in vitro and in vivo growth are certainly expected. In particular, the effective CA concentration in the virion is much higher than the highest concentrations that could be used in the HS-AFM experiments. In addition, several aspects of 3D growth of the capsid in the maturing virion are absent during $2 \mathrm{D}$ growth on a planar template: (i) the intrinsic curvature of the CA lattice observed in solution; (ii) the curvature induced by a $3 \mathrm{D}$ template (the negatively charged RNP core) instead of a flat 2D template (the negatively charged mica substrate); and (iii) the insertion 
of pentameric "defects" at defined positions that allows capsid closure and define capsid shape. Regarding intrinsic curvature, assembly of a viral capsid protein into a flat hexagonal lattice could result in accumulation of elastic stress from curvature mismatch, affecting the kinetics of the reaction. ${ }^{74}$ It may also have an effect on the average size of the patches being formed, even though for low concentrations of CA we found relatively large patch sizes suggesting that curvature mismatch could be counteracted by the mica substrate (Figure 2). Observation by HS-AFM of CA self-assembly on a curved surface will likely provide clues on the effect of the intrinsic curvature of a viral capsid protein lattice on the self-assembly reaction. It may also enable single-molecule studies on the insertion of pentameric "defects" on a curved hexagonal lattice.

To what extent capsid assembly in the maturing HIV virion is templated by the RNP core is unclear. It has been shown that the RNP is not strictly required for mature HIV capsid assembly. ${ }^{25}$ However, in the absence of the RNA-binding nucleocapsid protein, only some virions showed a normal morphology and contained a closed conical capsid. ${ }^{25}$ Accordingly, in in vitro studies where no template was present, capsid assembly required nonphysiological ionic strength ${ }^{59}$ unless crowding agents were added, ${ }^{60}$ and open-end tubes were, in general, largely dominant over cone-shaped capsid-like particles. $^{59,60}$ Thus, although the CA protein contains the information needed for capsid formation, the RNP core may act as a template for promoting the efficient assembly of the CA capsid in the maturing HIV virion.

Our approach to visualize the first steps of mature HIV capsid formation through assembly of a regular protein lattice, unlike other experimental $^{59,60}$ or theoretical ${ }^{66-70}$ approaches, has included an assembly template, the mica surface. The negative charge density of this template qualitatively mimics the negative charge of the nucleoprotein complex inside the virion. We have shown that during templated assembly of the CA lattice several oligomeric nuclei were formed, which diffused over the surface, became immobilized, and started to grow into independent patches. In addition, it was shown that the number of nuclei per unit area increased with total [CA] and that the time required for merging two growing patches was dependent on the relative orientation of their protein lattices. Given the very high effective $[\mathrm{CA}]$ inside the maturing virion, the formation of not one but several CA oligomeric nuclei inside the virion is a possibility to be considered. Some of those nuclei could grow into small CA patches that would never merge with other patches due to wrong orientation and/ or depletion of available CA subunits in the virion. These small CA oligomeric patches together with free CA subunits could account for the large fraction (roughly 50\%) of CA molecules inside the maturing virion that are not incorporated into the mature capsid. Moreover, the capsid itself could be built from more than one nucleation point moving on the RNP surface. These nuclei would give rise to a few independent CA lattice patches that would curve over the RNP template. These patches would eventually merge into a complete, closed capsid after their lattices are geometrically matched through diffusion, additional subunits are bound at the interfaces between them, and CA pentameric "defects" are included. This possibility requires experimental verification. However, the observation that in some HIV virions two mature capsids are formed that could grow from different nuclei is consistent with this proposal.

\section{CONCLUSIONS}

The minimalist HIV capsid-based system analyzed here was used as a model to experimentally unveil, in real time, possible pathways followed by single molecules during nucleation and subsequent growth of a protein-based supramolecular lattice. The results provide a detailed view on the dynamics of a supramolecular self-assembly process at the single molecule level. The presented real-time imaging of single molecules reversibly binding to the growing lattice provides direct evidence for stochastic pathways during assembly. The findings relate to the first steps of templated capsid assembly in the maturing HIV virion and provide insights that could support the development of molecular therapeutics and the bottom-up design and fabrication of nanodevices and nanomaterials.

\section{METHODS}

Protein Expression and Purification. Capsid protein CA of HIV type 1 (strain BH10) was expressed in E. coli BL21(DE3) cells that had been transfected with a recombinant plasmid pET21b(+) containing the full-length CA-coding sequence and purified following a previously described procedure. ${ }^{60}$ Briefly, cell cultures were grown at $37^{\circ} \mathrm{C}$, and protein expression was induced by addition of $1 \mathrm{mM}$ IPTG and incubation for $3 \mathrm{~h}$. Cells were harvested and frozen at -20 ${ }^{\circ} \mathrm{C}$ for storage. The cells were thawed and resuspended in $50 \mathrm{mM}$ Tris- $\mathrm{HCl} / 5 \mathrm{mM} \beta$-mercaptoethanol at $\mathrm{pH} 8$ and lysed by sonication. After centrifugation, CA protein in the soluble fraction was purified to near homogeneity by ammonium sulfate fractionation, ion-exchange chromatography in SP-Sepharose, and size-exclusion chromatography in Superdex 75 (all media from Amersham Biotech). The solution of purified CA thus obtained was thoroughly dialyzed against phosphate buffered saline (PBS) and stored at $-80{ }^{\circ} \mathrm{C}$ until use. Concentration and purity of CA protein stocks were respectively assessed by UVspectrophotometry and polyacrylamide gel electrophoresis.

HS-AFM Imaging of HIV Capsid Protein Lattice SelfAssembly. The AFM images were acquired in amplitude modulation tapping mode in liquid using a high-speed atomic force microscope (RIBM, Japan). Short cantilevers $(\sim 7 \mu \mathrm{m})$ with a nominal spring constant of $0.15 \mathrm{~N} / \mathrm{m}$ were used (USC-F1.2-k0.15, NanoWorld, Switzerland). The applied imaging forces were reduced by using a small set-point amplitude of $0.8 \mathrm{~nm}$ (for a $1 \mathrm{~nm}$ free amplitude) and constantly controlling the feedback parameters during imaging.

Assembly of the HIV capsid protein lattice from free CA molecules in phosphate-buffered saline (PBS) to approximate physiological conditions of $\mathrm{pH}$ and ionic strength was performed on a freshly cleaved mica substrate under the AFM microscope. Imaging was started on the mica surface in PBS. Next, a concentrated CA solution was added to reach the desired final protein concentration in the AFM liquid chamber. The protein solution was gently pipetted using a volume that was less than $10 \%$ of the total volume of liquid in the AFM chamber $(\sim 100 \mu \mathrm{L})$. The assembly reaction was triggered by the presence of the negatively charged mica substrate. ${ }^{61}$ Total $[\mathrm{CA}]$ did not exceed $20 \mu \mathrm{M}$ because higher concentrations prevented highresolution HS-AFM imaging. Images were primarily processed using built-in scripts (RIBM, Japan) in Igor Pro (Wavemetrics, Lake Oswego, OR, USA) and analyzed using ImageJ software. The images/ movies were corrected minimally for tilt, drift, and contrast. Unless otherwise mentioned, the times reported in AFM/images/videos are relative. Imaging was disrupted during the initial addition and mixing of the proteins, which typically took $\sim 10 \mathrm{~s}$. This time was relatively small compared to the time needed for the CA molecules to reach the surface by diffusion and become adsorbed.

\section{ASSOCIATED CONTENT}

\section{Supporting Information}

The Supporting Information is available free of charge at https://pubs.acs.org/doi/10.1021/acsnano.0c03207. 
Figure S1, structural analysis of HIV capsid protein 2D lattice visualized by HS-AFM; Figure S2, surface crowding and CA protein oligomerization on substrate; Figure S3, isotropic diffusion of CA protein oligomers prior to immobilization on substrate; Figure S4, surface growth of capsid protein patches; Figure S5, growth dynamics of individual CA hexamers at edge of capsid protein patch; Figure S6, force-induced disassembly of capsid protein patch; and Figure S7, differences between individual association and dissociation events at early or late stages during assembly of capsid protein lattice (PDF)

Movie 1, fluctuations in position of individual CA subunits in capsid protein lattice imaged by HS-AFM (AVI)

Movie 2, nucleation and fusion of capsid protein patches (AVI)

Movie 3, gradual assembly of CA hexamer at edge of capsid protein patch (AVI)

Movie 4, association and/or dissociation events at edge of capsid protein patch (AVI)

Movie 5, disassembly of CA hexamers at edge of capsid protein patch (AVI)

Movie 6, dissociation of hexamer-sized CA subunits followed by binding to nearby protein patch (AVI)

\section{AUTHOR INFORMATION}

\section{Corresponding Authors}

Mauricio G. Mateu - Centro de Biología Molecular "Severo Ochoa", Universidad Autonoma de Madrid, 28049 Madrid, Spain; 이이이.org/0000-0002-2915-1529; Email: mgarcia@cbm.csic.es

Wouter H. Roos - Moleculaire Biofysica, Zernike Instituut, Rijksuniversiteit Groningen, 9712 CP Groningen, The Netherlands; 이이이.org/0000-0002-5104-0139; Email: w.h.roos@rug.nl

\section{Authors}

Alejandro Valbuena - Centro de Biología Molecular "Severo Ochoa", Universidad Autonoma de Madrid, 28049 Madrid, Spain

Sourav Maity - Moleculaire Biofysica, Zernike Instituut, Rijksuniversiteit Groningen, 9712 CP Groningen, The

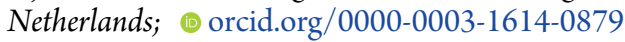

Complete contact information is available at:

https://pubs.acs.org/10.1021/acsnano.0c03207

\section{Author Contributions \\ ${ }^{\S}$ A.V. and S.M. contributed equally to this work. \\ Notes}

The authors declare no competing financial interest.

\section{ACKNOWLEDGMENTS}

We gratefully acknowledge E. Sahagún (Scixel company) for help with the design of the Table of Contents graphic and Figure 5 and D. Reguera (University of Barcelona) for critical reading of the manuscript. S.M. was the recipient of a MSCA Individual fellowship (INTERACT 751404). M.G.M. is an associate member of the Institute for Biocomputation and Physics of Complex Systems, Zaragoza, Spain. This work was funded by grants from MICINN/FEDER EU (Spain, BIO2015-69928-R and RTI2018-096635-B-100) to M.G.M. and a VIDI grant from the Nederlandse Organisatie voor Wetenschappelijk Onderzoek and a STW-Perspectief grant "Cancer-ID" to W.H.R. A travel grant to A.V. by the European ARBRE-Mobieu Consortium (COST Action CA15126) is acknowledged.

\section{REFERENCES}

(1) Agbandje-McKenna, M., McKenna, R., Eds.; Structural Virology; Royal Society of Chemistry Publishing: Cambridge, 2011; DOI: $10.1039 / 9781849732239$.

(2) Rossmann, M. G., Rao, V. B., Eds.; Viral Molecular Machines; Springer: New York, 2012; DOI: 10.1007/978-1-4614-0980-9.

(3) Mateu, M. G., Ed.; Structure and Physics of Viruses; Springer: Dordrecht, 2013; DOI: 10.1007/978-94-007-6552-8.

(4) Porterfield, J. Z.; Zlotnick, A. An Overview of Capsid Assembly Kinetics. In Physical Virology; Stockley, P. G., Twarock, R., Eds.; Imperial College Press: London, 2010; pp 131-158, DOI: 10.1142/ 97818481646660006.

(5) Zlotnick, A.; Fane, B. A. Mechanisms of Icosahedral Virus Assembly. In Structural Virology; Agbandje-McKenna, M., McKenna, R., Eds.; Royal Society of Chemistry Publishing, Cambridge, 2011; pp 180-202, DOI: 10.1039/9781849732239-00180.

(6) Luque, A.; Reguera, D. Theoretical Studies on Assembly, Physical Stability and Dynamics of Viruses. In Structure and Physics of Viruses; Mateu, M. G., Ed.; Springer: Dordrecht, 2013; pp 553-595, DOI: $10.1007 / 978-94-007-6552-819$.

(7) Mateu, M. G. Assembly, Stability and Dynamics of Virus Capsids. Arch. Biochem. Biophys. 2013, 531, 65-79.

(8) Hagan, M. F. Modeling Viral Capsid Assembly. Adv. Chem. Phys. 2014, 155, 1-68.

(9) Perlmutter, J. D.; Hagan, M. F. Mechanisms of Virus Assembly. Annu. Rev. Phys. Chem. 2015, 66, 217-239.

(10) Hagan, M. F.; Zandi, R. Recent Advances in Coarse-Grain Modeling of Virus Assembly. Curr. Opin. Virol. 2016, 18, 36-43.

(11) Prevelige, P. E., Jr. Inhibiting Virus-Capsid Assembly by Altering the Polymerisation Pathway. Trends Biotechnol. 1998, 16, $61-65$.

(12) Zlotnick, A.; Mukhopadhyay, S. Virus Assembly. Trends Microbiol. 2011, 19, 14-23.

(13) Douglas, T.; Young, M. Viruses: Making Friends with Old Foes. Science 2006, 312, 873-875.

(14) Koudelka, K. J.; Pitek, A. S.; Manchester, M.; Steinmetz, N. F. Virus-Based Nanoparticles as Versatile Nanomachines. Annu. Rev. Virol. 2015, 2, 379-401.

(15) Mateu, M. G. Assembly, Engineering and Applications of VirusBased Protein Nanoparticles. Adv. Exp. Med. Biol. 2016, 940, 83-120.

(16) Raja, I. S.; Kim, C.; Song, S. J.; Shin, Y. C.; Kang, M. S.; Hyon, S. H.; Oh, J. W.; Han, D. W. Virus-Incorporated Biomimetic Nanocomposites for Tissue Regeneration. Nanomaterials 2019, 9, 1014.

(17) Dufort, C. C.; Dragnea, B. Bio-Enabled Synthesis of Metamaterials. Annu. Rev. Phys. Chem. 2010, 61, 323-344.

(18) Gerrard, J. A., Ed. Protein Nanotechnology, 2nd ed.; Humana Press: New York, 2013; DOI: 10.1007/978-1-62703-354-1.

(19) Wegst, U. G.; Bai, H.; Saiz, E.; Tomsia, A. P.; Ritchie, R. O. Bioinspired Structural Materials. Nat. Mater. 2015, 14, 23-36.

(20) Cortajarena, A. L., Grove, T. Z., Eds.; Protein-Based Engineered Nanostructures; Springer: Switzerland, 2016; DOI: 10.1007/978-3319-39196-0.

(21) Fersht, A. R., Ed.; Structure and Mechanism in Protein Science; World Scientific: Singapore, 2017; DOI: 10.1142/10574.

(22) Perilla, J. R.; Gronenborn, A. M. Molecular Architecture of the Retroviral Capsid. Trends Biochem. Sci. 2016, 41, 410-420.

(23) Zhao, G.; Perilla, J. R.; Yufenyuy, E. L.; Meng, X.; Chen, B.; Ning, J.; Ahn, J.; Gronenborn, A. M.; Schulten, K.; Aiken, C.; Zhang, P. Mature HIV-1 Capsid Structure by Cryo-Electron Microscopy and All-Atom Molecular Dynamics. Nature 2013, 497, 643-646. 
(24) Ganser, B. K.; Li, S.; Klishko, V. Y.; Finch, J. T.; Sundquist, W. I. Assembly and Analysis of Conical Models for the HIV-1 Core. Science 1999, 283, 80-83.

(25) Mattei, S.; Flemming, A.; Anders-Össwein, M.; Kräusslich, H. G.; Briggs, J. A.; Muller, B. RNA and Nucleocapsid Are Dispensable for Mature HIV-1 Capsid Assembly. J. Virol. 2015, 89, 9739-9747.

(26) Rapaport, D. C. Molecular Dynamics Simulation of Reversibly Self-Assembling Shells in Solution Using Trapezoidal Particles. Phys. Rev. E 2012, 86, 51917-51924.

(27) Twarock, R.; Stockley, P. G. RNA-Mediated Virus Assembly: Mechanisms and Consequences for Viral Evolution and Therapy. Annu. Rev. Biophys. 2019, 48, 495-514.

(28) Sorger, P. K.; Stockley, P. G.; Harrison, S. C. Structure and Assembly of Turnip Crinkle Virus. II. Mechanism of Reassembly In Vitro. J. Mol. Biol. 1986, 191, 639-658.

(29) Prevelige, P. E., Jr.; Thomas, D.; King, J. Nucleation and Growth Phases in the Polymerization of Coat and Scaffolding Subunits into Icosahedral Procapsid Shells. Biophys. J. 1993, 64, 824835.

(30) Medrano, M.; Fuertes, M. A.; Valbuena, A.; Carrillo, P. J. P.; Rodríguez-Huete, A.; Mateu, M. G. Imaging and Quantitation of A Succession of Transient Intermediates Reveal the Reversible SelfAssembly Pathway for a Simple Icosahedral Virus Capsid. J. Am. Chem. Soc. 2016, 138, 15385-15396.

(31) Li, C.; Kneller, A. R.; Jacobson, S. C.; Zlotnick, A. Single Particle Observation of SV40 VP1 Polyanion-Induced Assembly Shows That Substrate Size and Structure Modulate Capsid Geometry. ACS Chem. Biol. 2017, 12, 1327-1334.

(32) Teschke, C. M.; Parent, K. N. Let the Phage Do the Work: Using the Phage P22 Coat Protein Structures as a Framework to Understand Its Folding and Assembly Mutants. Virology 2010, 401, 119-130.

(33) Tuma, R.; Tsuruta, H.; French, K. H.; Prevelige, P. E., Jr. Detection of Intermediates and Kinetic Control during Assembly of Bacteriophage P22 Procapsid. J. Mol. Biol. 2008, 381, 1395-1406.

(34) Tresset, G.; Le Coeur, C.; Bryche, J.-F.; Tatou, M.; Zeghal, M.; Charpilienne, A.; Poncet, D.; Constantin, D.; Bressanelli, S. Norovirus Capsid Proteins Self-Assemble through Biphasic Kinetics via LongLived Stave-Like Intermediates. J. Am. Chem. Soc. 2013, 135, 1537315381.

(35) Law-Hine, D.; Zeghal, M.; Bressanelli, S.; Constantin, D.; Tresset, G. Reconstruction of the Disassembly Pathway of an Icosahedral Viral Capsid and Shape Determination of Two Successive Intermediates. Soft Matter 2016, 12, 6728-6736.

(36) Asor, R.; Selzer, L.; Schlicksup, C. J.; Zhao, Z.; Zlotnick, A.; Raviv, U. Assembly Reactions of Hepatitis B Capsid Protein into Capsid Nanoparticles Follow a Narrow Path through a Complex Reaction Landscape. ACS Nano 2019, 13, 7610-7626.

(37) Chevreuil, M.; Law-Hine, D.; Chen, J.; Bressanelli, S.; Combet, S.; Constantin, D.; Degrouard, J.; Möller, J.; Zeghal, M.; Tresset, G. Nonequilibrium Self-Assembly Dynamics of Icosahedral Viral Capsids Packaging Genome or Polyelectrolyte. Nat. Commun. 2018, 9, 3071.

(38) Panahandeh, S.; Li, S.; Marichal, L.; Leite Rubim, R.; Tresset, G.; Zandi, R. How a Virus Circumvents Energy Barriers to Form Symmetric Shells. ACS Nano 2020, 14, 3170-3180.

(39) Shoemaker, G. K.; Van Duijn, E.; Crawford, S. E.; Uetrecht, C.; Baclayon, M.; Roos, W. H.; Wuite, G. J. L.; Estes, M. K.; Prasad, B. V. V.; Heck, A. J. Norwalk Virus Assembly and Stability Monitored by Mass Spectrometry. Mol. Cell. Proteomics 2010, 9, 1742-1751.

(40) Uetrecht, C.; Barbu, I. M.; Shoemaker, G. K.; Van Duijn, E.; Heck, A. J. Interrogating Viral Capsid Assembly with Ion MobilityMass Spectrometry. Nat. Chem. 2011, 3, 126-132.

(41) Lutomski, C. A.; Lyktey, N. A.; Pierson, E. E.; Zhao, Z.; Zlotnick, A.; Jarrold, M. F. Multiple Pathways in Capsid Assembly. J. Am. Chem. Soc. 2018, 140, 5784-5790.

(42) Ashcroft, A. E. Mass Spectrometry Studies of Virus Assembly. Curr. Opin. Virol. 2019, 36, 17-24.
(43) Harms, Z. D.; Selzer, L.; Zlotnick, A.; Jacobson, S. C. Monitoring Assembly of Virus Capsids with Nanofluidic Devices. ACS Nano 2015, 9, 9087-9096.

(44) Garmann, R. F.; Goldfain, A. M.; Manoharan, V. N. Measurements of the Self-Assembly Kinetics of Individual Viral Capsids around Their RNA Genome. Proc. Natl. Acad. Sci. U. S. A. 2019, 116, 22485-22490.

(45) Borodavka, A.; Tuma, R.; Stockley, P. G. Evidence that Viral RNAs have Evolved for Efficient, Two-Stage Packaging. Proc. Natl. Acad. Sci. U. S. A. 2012, 109, 15769-15774.

(46) Marchetti, M.; Kamsma, D.; Cazares Vargas, E.; Hernandez Garcia, A.; Van Der Schoot, P.; De Vries, R.; Wuite, G. J. L.; Roos, W. H. Real-Time Assembly of an Artificial Virus Elucidated at the SingleParticle Level. Nano Lett. 2019, 19, 5746-5753.

(47) Van Rosmalen, M. G. M.; Kamsma, D.; Biebricher, A. S.; Li, C.; Zlotnick, A.; Roos, W. H.; Wuite, G. J. L. Revealing in Real-Time a Multistep Assembly Mechanism for SV40 Virus-Like Particles. Sci. Adv. 2020, 6, eaaz1639.

(48) Ando, T. High-Speed Atomic Force Microscopy Coming of Age. Biophys. Rev. 2017, 9, 421-429.

(49) Kodera, N.; Yamamoto, D.; Ishikawa, R.; Ando, T. Video Imaging of Walking Myosin $\mathrm{V}$ by High-Speed Atomic Force Microscopy. Nature 2010, 468, 72-76.

(50) Uchihashi, T.; Lino, R.; Ando, T.; Noji, H. High-Speed Atomic Force Microscopy Reveals Rotary Catalysis of Rotorless F1-ATPase. Science 2011, 333, 755-758.

(51) Preiner, J.; Horner, A.; Karner, A.; Ollinger, N.; Siligan, C.; Pohl, P.; Hinterdorfer, P. High-Speed AFM Images of Thermal Motion Provides Stiffness Map of Interfacial Membrane Protein Moieties. Nano Lett. 2015, 15, 759-763.

(52) Miyagi, A.; Chipot, C.; Rangl, M.; Scheuring, S. High-Speed Atomic Force Microscopy Shows That Annexin V Stabilizes Membranes on the Second Timescale. Nat. Nanotechnol. 2016, 11, 783-790.

(53) Bertin, A.; de Franceschi, N.; de la Mora, E.; Maiti, S.; Alqabandi, M.; Miguet, N.; di Cicco, A.; Roos, W. H.; Mangenot, S.; Weissenhorn, W.; Bassereau, P. Human ESCRT-III Polymers Assemble on Positively Curved Membranes and Induce Helical Membrane Tube Formation. Nat. Commun. 2020, 11, 2663.

(54) Sutter, M.; Faulkner, M.; Aussignargues, C.; Paasch, B. C.; Barrett, S.; Kerfeld, C. A.; Liu, L.-N. Visualization of Bacterial Microcompartment Facet Assembly Using High-Speed Atomic Force Microscopy. Nano Lett. 2016, 16, 1590-1595.

(55) Banerjee, S.; Sun, Z.; Hayden, E. Y.; Teplow, D. B.; Lyubchenko, Y. L. Nanoscale Dynamics of Amyloid $\beta-42$ Oligomers as Revealed by High-Speed Atomic Force Microscopy. ACS Nano 2017, 11, 12202-12209.

(56) Nievergelt, A. P.; Banterle, N.; Andany, S. H.; Gönczy, P.; Fantner, G. E. High-Speed Photothermal Off-Resonance Atomic Force Microscopy Reveals Assembly Routes of Centriolar Scaffold Protein Sas-6. Nat. Nanotechnol. 2018, 13, 696-701.

(57) Maity, S.; Caillat, C.; Miguet, N.; Sulbaran, G.; Effantin, G.; Schoehn, G.; Roos, W. H.; Weissenhorn, W. VPS4 Triggers Constriction and Cleavage of ESCRT-III Helical Filaments. Sci. Adv. 2019, 5, No. eaau7198.

(58) Ganser-Pornillos, B. K.; Cheng, A.; Yeager, M. Structure of Full-Length HIV-1 CA: A Model for the Mature Capsid Lattice. Cell 2007, 131, 70-79.

(59) Lanman, J.; Sexton, J.; Sakalian, M.; Prevelige, P. E., Jr. Kinetic Analysis of the Role of Intersubunit Interactions in Human Immunodeficiency Virus Type 1 Capsid Protein Assemby In Vitro. J. Virol. 2002, 76, 6900-6908.

(60) Del Alamo, M.; Rivas, G.; Mateu, M. G. Effect of Macromolecular Crowding Agents on Human Immunodeficiency Virus Type 1 Capsid Protein Assembly In Vitro. J. Virol. 2005, 79, 14271-14281.

(61) Valbuena, A.; Mateu, M. G. Quantification and Modification of the Equilibrium Mechanics and Dynamics of a Viral Capsid Lattice 
Self-Assembled as a Protein Nanocoating. Nanoscale 2015, 7, 1495314964.

(62) Valbuena, A.; Mateu, M. G. Kinetics of Surface-Driven SelfAssembly and Fatigue-Induced Disassembly of a Virus-Based Nanocoating. Biophys. J. 2017, 112, 663-673.

(63) Johnson, J. M.; Tang, J.; Nyame, Y.; Willits, D.; Young, M. J.; Zlotnick, A. Regulating Self-Assembly of Spherical Oligomers. Nano Lett. 2005, 5, 765-770.

(64) Zandi, R.; van der Schoot, P.; Reguera, D.; Kegel, W.; Reiss, H. Size Classic Nucleation Theory of Virus Capsids. Biophys. J. 2006, 90, 1939-1948.

(65) Tsiang, M.; Niedziela-Majka, A.; Hung, M.; Jin, D.; Hu, E.; Yant, S.; Samuel, D.; Liu, X.; Sakowicz, R. A Trimer of Dimers Is the Basic Building Block for Human Immunodeficiency Virus-1 Capsid Assembly. Biochemistry 2012, 51, 4416-4428.

(66) Chen, B.; Tycko, R. Simulated Self-Assembly of the HIV-1 Capsid: Protein Shape and Native Contacts Are Sufficient for TwoDimensional Lattice Formation. Biophys. J. 2011, 100, 3035-3044.

(67) Grime, J. M. A.; Voth, G. A. Early Stages of the HIV-1 Capsid Protein Lattice Formation. Biophys. J. 2012, 103, 1774-1783.

(68) Chen, B. HIV Capsid Assembly, Mechanism and Structure. Biochemistry 2016, 55, 2539-2552.

(69) Grime, J. M. A.; Dama, J. F.; Ganser-Pornillos, B. K.; Woodward, C. L.; Jensen, G. J.; Yeager, M.; Voth, G. A. CoarseGrained Simulation Reveals Key Features of HIV-1 Capsid Assembly. Nat. Commun. 2016, 7, 11568.

(70) Pak, A. J.; Grime, J. M. A.; Yu, A.; Voth, G. A. Off Pathway Assembly: A Broad-Spectrum Mechanism of Action for Drugs That Undermine Controlled HIV-1 Viral Capsid Formation. J. Am. Chem. Soc. 2019, 141, 10214-10224.

(71) Gamble, T. R.; Yoo, S.; Vajdos, F. F.; Von Schwedler, U. K.; Worthylake, D. K.; Wang, H.; McCutcheon, J. P.; Sundquist, W. I.; Hill, C. P. Structure of the Carboxy-Terminal Dimerization Domain of the HIV-1 Capsid Protein. Science 1997, 278, 849-853.

(72) Miles, P.; Frankel, D. Lipid Directed Assembly of the HIV Capsid Protein. Soft Matter 2014, 10, 9562-9567.

(73) Bocanegra, R.; Alfonso, C.; Rodríguez-Huete, A.; Fuertes, M. A.; Jiménez, M.; Rivas, G.; Mateu, M. G. Association Equilibrium of the HIV-1 Capsid Protein in a Crowded Medium Reveals that Hexamerization during Capsid Assembly Requires a Functional CDomain Dimerization Interface. Biophys. J. 2013, 104, 884-893.

(74) Wagner, J.; Zandi, R. The Robust Assembly of Small Symmetric Nanoshells. Biophys. J. 2015, 109, 956-965. 